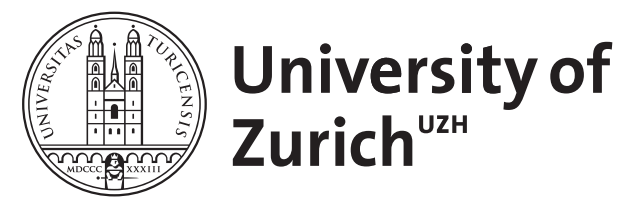

\title{
Depression in autoimmune diseases
}

\author{
Pryce, Christopher R ; Fontana, Adriano
}

\begin{abstract}
Up to $50 \%$ of patients with autoimmune diseases show an impairment of health-related quality of life and exhibit depression-like symptoms. The immune system not only leads to inflammation in affected organs, but also mediates behavior abnormalities including fatigue and depression-like symptoms. This review focuses on the different pathways involved in the communication of the immune system with the neuronal network and the body's timing system. The latter is built up by a hierarchically organized expression of clock genes. As discussed here, the activation of the immune system interferes with high amplitude expression of clock genes, an effect which may play a pivotal role in depression-like behavior in autoimmune diseases.
\end{abstract}

DOI: https://doi.org/10.1007/7854_2016_7

Posted at the Zurich Open Repository and Archive, University of Zurich

ZORA URL: https://doi.org/10.5167/uzh-125882

Book Section

Accepted Version

Originally published at:

Pryce, Christopher R; Fontana, Adriano (2016). Depression in autoimmune diseases. In: Current Topics in Behavioral Neurosciences. Cham: Springer, 139-154.

DOI: https://doi.org/10.1007/7854_2016_7 


\section{Depression in Autoimmune Diseases}

\section{Christopher R. Pryce (1) and Adriano Fontana ${ }^{\left(2,{ }^{*}\right)}$}

(1) Preclinical Laboratory for Translational Research into Affective Disorders, Department of Psychiatry, Psychotherapy \& Psychosomatics, Psychiatric Hospital, University of Zurich

(2) Institute of Experimental Immunology, University of Zurich, Zurich, Switzerland

$\left(^{*}\right)$ Corresponding author: Institute of Experimental Immunology, University of Zurich, Winterthurerstrasse 190, CH-8057 Zürich, Switzerland. email address: fontana.adriano7@gmail.com

Key words: Systemic lupus erythematosus; Rheumatoid arthritis; Hepatitis, Tumor necrosis factor; Interleukin-1, Twist1; Indoleamine 2-3, dioxygenase, Kynurenine

\section{Summary}

Up to $50 \%$ of patients with autoimmune diseases show an impairment of healthrelated quality of life and exhibit depression-like symptoms. The immune system not only leads to inflammation in affected organs, but also mediates behavior abnormalities including fatigue and depression-like symptoms. This review focuses on the different pathways involved in the communication of the immune system with the neuronal network and the body's timing system. The latter is built up by a hierarchically organized expression of clock genes. As discussed here, the activation of the immune system interferes with high amplitude expression of clock genes, an effect which may play a pivotal role in depressionlike behavior in autoimmune diseases.

\section{Introduction}


In autoimmune diseases, psychological symptoms, such as fatigue, loss of interest in daily activities, and cognitive deficits, are common. Whilst these symptoms are often described as sickness behaviour, they are also core symptoms in the diagnosis of depression. This raises a number of issues, and perhaps most important is the issue of whether sickness behaviour and depression are one and the same, or do they have fundamentally different pathophysiologies so that it is more appropriate to regard them as separate clinical states? The present chapter reviews the evidence for inflammation in depression and for depression-like symptoms in autoimmune diseases. It is not currently possible to unequivocally answer the issues raised above. However, we do provide an overview of some of the most relevant evidence that can inform this controversial, interesting and medically important debate.

Patients with depression, most notably major depressive disorder (MDD), who are otherwise medically healthy have been found to exhibit features of immune activation. Increases of the circulating cytokines interleukin (IL)-6 and tumor necrosis factor alpha (TNF), their soluble receptors, and the acute phase protein, C-reactive protein (CRP), have been reported (Dowlati et al., 2010); for review see: (Felger and Lotrich, 2013; Miller et al., 2009). Moreover, gene expression profiling conducted on post-mortem brain tissue samples from the frontal cortex of patients with MDD shows an upregulated expression of cytokines including eg IL-1 $\beta$, IL-18, IL-8, IL-12, lymphotoxin alpha and interferon (IFN)- $\gamma$ (Shelton et al., 2011). More direct evidence for the involvement of cytokines in the pathophysiology of MDD comes from the observations that (i) anti-TNF treatment has therapeutic effects in resistant MDD with increased CRP (Raison et al., 2013), and (ii) the development of depressed mood, anxious symptoms and impaired cognition in human patients treated with type I interferons ((Miller et al., 2009)). Likewise, in mice, the systemic or intracerebroventricular administration of cytokines or cytokine inducers leads to depressive-like behavior (Fu et al., 2010). Pathways that translate the cytokine signals from the periphery to the brain involve (i) actions of cytokines on the vagal nerve and activation of Toll-like receptors in circumventricular organs, (ii) interactions of cytokines with the hypothalamic-pituitary-adrenal axis, and (iii) cytokine 
triggered activation of indoleamine-2,3-dioxygenase leading to tryptophan catabolism and decreased serotonin concentrations in the central nervous system (CNS) (for review see (Dantzer et al., 2008; Haroon et al., 2012).

In light of the putative causal link of increased cytokines and depression, we may ask whether depression-like symptoms or depression per se is also a hallmark in autoimmune diseases. The latter are characterized by anti-self $\mathrm{T}$ - and B-cell reactivity, which leads to organ dysfunction due to destruction of parenchymal cells in various organs, including kidney, lung, liver, intestinal tract and the peripheral and central nervous systems. The immune response towards autoantigens is characterized by activation of neutrophils, monocytesmacrophages, dendritic cells and lymphocytes on the one hand, and decreased activity of negative feedback loops provided by regulatory T- and B- cells and by suppressive macrophages, on the other. Both the priming and effector phases of the autoimmune response are associated with increased production of cytokines. Since inducers of cytokines and cytokines per se induce depressive-like behavior in both experimental animal models and humans it is not surprising that autoimmune diseases have also been associated with depression. In the following we will focus on the evidence for depression-like symptoms in autoimmune diseases and animal models thereof. We will concentrate on systemic lupus erythematosus (SLE) and rheumatoid arthritis (RA), since in these diseases large cohort studies on mental health have been conducted and therefore a relatively amount of data are available. However, depression-like symptoms have also been well described in many other autoimmune diseases including multiple sclerosis and inflammatory bowel disease (for review see (Greenley et al., 2010; Marrie et al., 2015)). Moreover, depression-like symptoms are also a hallmark of autoinflammatory diseases. These inherited diseases are characterized by inadequate activation of the immune system with production of cytokines due to mutations in genes encoding for the inflammasome. The most frequent form is familial Mediterranean fever (FMF), which develops due to mutations of the pyrin/FMF gene. In FMF patients depression-like behavior is seen frequently; the depression-like symptom severity does not depend on FMF duration, but rather on the FMF severity score (Makay et al., 2010). 


\section{Depression-like symptoms in systemic lupus erythematosus}

Systemic lupus erythematosus (SLE) is an autoimmune disease characterized by loss of tolerance, which is associated with production of autoantibodies and immune-mediated injury resulting in tissue damage in various organs including kidney, lung, skin and central nervous system. Activation of the complement system by immune complexes leads to endothelial cell disruption. SLE takes a relapsing-remitting disease course which, depending on the affected organ can be mild or severe. Numerous studies show that patients with SLE have a significantly poorer health-related quality of life (HRQoL) and exhibit depression-like symptoms more often than do healthy controls. In a systematic literature search 17 studies were identified that reported on depression comorbidity symptoms, with prevalence a rate of $17-75 \%$ depending on study (Palagini et al., 2013). Different methods were used to assess depression across these studies, and this is likely to contribute to the wide range in the prevalence reported.

A study on 170 SLE patients found HRQoL to be negatively influenced mainly by disease activity and depression (Shen et al., 2014). Both parameters were associated with higher levels of pain and fatigue (Pettersson et al., 2012; Waldheim et al., 2013). Using the Beck Depression Inventory (BDI) patients with SLE had a higher score compared with the general population from a pan-Europe population based study (van Exel et al., 2013). The prevalence of depression was found to be $16.6 \%$ in SLE and $6.7 \%$ in controls. There was no association between disease activity and organ damage. Likewise in 113 patients with SLE no relationship between HRQoL and SLE activity or disease duration was observed (Yilmaz-Oner et al., in press). In 110 lupus patients the SLE activity index did correlate with the anxiety score, but not with depression (Tay et al., 2015). Another study using BDI-II showed 53 of 127 SLE patients (41.7\%) to have moderate or severe depressive symptoms (Karol et al., 2013). When assessing sleep behavior in 81 patients with SLE, depressive symptoms were associated significantly with poor sleep quality, and cognitive deficits occurred in 
16 of 82 patients (Palagini et al., 2014). Impaired cognitive performance in sustained attention and spatial working memory tests was related to SLE, but not to depression (Calderon et al., 2014). With respect to the important question of whether depression-like symptoms in autoimmune-disease patients results in poorer disease control, in general it is indeed thought that depression is associated with increased disease activity, decreased medication adherence, and decreased work productivity (Bachen et al., 2009; Panopalis et al., 2008).

Approximately 15-20\% of SLE cases begin in childhood, at a median age of onset of 12-13 years. In children and adolescents with SLE $(n=38)$ and young adults with childhood-onset of disease $(n=16)$, depression was observed in $26 \%$ and $44 \%$ of the patients, respectively (Ahola Kohut et al., 2013). Depression was not correlated with disease duration, HRQoL or SLE nephritis. In pediatric lupus and mixed connective tissue disease, depression was associated with poor disease control; non-Caucasian patients were at significantly higher risk for depressionlike symptoms than Caucasian patients (Knight et al., 2015; Knight et al., 2014). In general disease scores, lupus nephritis and involvement of the central nervous system - neuropsychiatric lupus (NPSLE) - takes a more aggressive course in African-Americans. In this group of patients, higher perceived racism was associated with having moderate to severe depression (Vina et al., 2015). Suicidal ideation has been observed in pediatric lupus with NPSLE in 34\% of 53 patients, and $20 \%$ of those with ideation attempted suicide (Lim et al., 2013). In adults with SLE, suicidal ideation is common in up to one-third of patients with and without NPSLE, and is associated with higher disease activity, increased depression and anxiety severity and previous suicide attempts (Karassa et al., 2003; Mok et al., 2014; Xie et al., 2012).

The mechanisms underlying depression or depression-like symptoms in SLE are not yet clear, but are likely to be multifactorial. The burden of having a chronic illness, the use of corticosteroids and immunosuppressive drugs for disease control and prevention of disease, microvasculopathy as well as the development of secondary cerebrovascular injury due to hypertension, hyperlipidemia and anti-phospholipid antibodies, may all constitute risk factors in SLE. Depression 
in women with SLE $(n=161)$ correlates with the presence of cardiovascular disease (Greco et al., 2012). Serum TNF was found to be higher in SLE $(n=54)$ compared to controls. Interestingly, increased TNF was associated with more severe depression-like symptoms as evaluated by the Hospital Anxiety and Depressive Scale (HADs) (Mak et al., 2013). Injection of TNF in rodents leads to sickness behavior and depression-like behavior (Fu et al., 2010). Patients with SLE exhibit high serum levels of IFN type I and overexpress IFN-I-stimulated genes in peripheral blood cells. The increased IFN-I signature is associated with active disease including lupus nephritis. Since in patients with multiple sclerosis or hepatitis $\mathrm{C}$, treatment with IFN- $\alpha$ induces depression and the risk of suicidal ideation, the endogenous expression of IFN-I in SLE may contribute to depression-like symptoms. However, in patients with SLE the increased IFN-I levels are found to not be correlated with depression and fatigue (Kellner et al., 2010).

Polymorphisms of the serotonin transporter gene promoter region (PR-5HTT) were studied in 96 SLE patients. Using the Hamilton depression rating scale (HDRS), depression scores were found to be associated with patients being homozygous for the short allele of the PR-5HTT gene (Xu et al., 2013). The methylation status of the region studied did not differ in patients with depression versus those without. In neuropsychiatric lupus, anti-ribosomal P antibodies $(\mathrm{Ab})$ have been reported to occur more frequently in SLE patients with depression (Karimifar et al., 2013). In patients with a disease duration of less than two years, depression severity was correlated with anti-ribosomal $\mathrm{P} \mathrm{Ab}$ levels. The antibody recognizes the carboxy 22 amino acids of the three large subunit ribosomal phosphoproteins, called P0, P1, and P2. Anti-ribosomal P Ab bind to neurons in the hippocampus, cingulate cortex, and the primary olfactory piriform cortex, and cause calcium influx and apoptosis (Bravo-Zehnder et al., 2015). Intracerebroventricular injection of anti-ribosomal P Ab in mice induces a depression-like behavior and olfactory impairment. Mice displayed impaired passive avoidance (Katzav et al., 2014). In the pathophysiology of NPSLE, antidouble stranded (ds)-DNA Ab may contribute to the involvement of the central nervous system. Some anti-native-DNA Ab cross-react with the GluN2A and 
GluN2B subunits of the N-methyl-D-aspartate (NMDA) receptor for the neurotransmitter glutamate (Chang et al., 2015). Based on mouse experiments, cross-reacting antibodies may contribute to the neurocognitive impairment and other symptoms of NPSLE. When injected with the 16/6 idiotypic antibodies, which recognize anti-ds-DNA Ab as well as brain glycoproteins and glycolipids, mice develop an impairment of spatial memory (Gono et al., 2013; Kivity et al., 2013).

\section{Rheumatoid arthritis and depression-like symptoms}

In addition to its symptoms in affected joints, rheumatoid arthritis (RA) is associated with substantial mental health problems. In particular, depression has been documented to contribute significantly to impaired HRQoL, unemployment and loss of work productivity. Depression is very common in RA, the prevalence reported to be 13 to $42 \%$ (Isik et al., 2007). As reviewed recently, depression in RA provides an independent risk factor for cardiovascular disease and myocardial infarction, suicidal ideation and death, even after controlling for RA disease duration, disease activity, disability and pain (Margaretten et al., 2011). Depression in RA patients is most common in females and in younger age groups (Fifield et al., 1996). The risk to develop depression is highest in the first five years after RA diagnosis (Wang et al., 2014). Whilst pain is likely to contribute to the development of depression, the link between the severity of measurable parameters of arthritis and depression is less stringent. Since cytokines play a pivotal role in the synovial inflammation in RA and TNF blockers exert strong therapeutic effects, it may be suggested that the cytokine - brain connection is essential in depression in RA. The hypothesis is supported by the observation of persistence of depression in patients with poor disease control by TNF blockers (Hider et al., 2009). However, studies investigating an association between depression in RA and increased CRP have yielded conflicting findings (Margaretten et al., 2011). In a recent study on RA ( $n=102$ with 75\% females), using the Beck depression index II (BDI II) to assess depression, and the Health assessment questionnaire (HAQ) and the Disease activity score (DAS) to quantitate RA activity, a correlation of severity of depression and RA activity was identified (Imran et al., 2015). Somewhat in line with these data is the finding 
that functional disability constitutes a significant risk factor for the occurrence of depression in RA (Katz and Yelin, 2001). Amelioration of depression symptoms in RA is linked to treatment-related RA control, positive psychological coping mechanisms, and social support (for review see (Margaretten et al., 2011)).

\section{Depression-like behavior in animal models of autoimmune diseases}

It is well established that rodents injected systemically or intacerebroventricularly with cytokines including TNF and IL-1 $\beta$ show an increase in sleep at times when mice are usually active (for review see (Imeri and Opp, 2009; Krueger, 2008)). NREM sleep was found to be increased in the dark phase of the $12 \mathrm{hr}$ light-dark circadian cycle. This effect is associated with increase in body temperature, loss of body weight and development of depression-like behavioral changes). The same clinical picture emerges when mice are treated with activators of Toll-like receptors including the synthetic analogs of ds-RNA, namely Poly(I:C), lipopolysaccharide (LPS) extracted from gram-negative bacteria, or peptidoglycans. These experimental animal models present with depression-like behavior due to activation of the innate immune system. Animal models of depression-like behavior that are based on activation of the acquired immune system are less well established. In the following sections we discuss two models which do indeed involve or mimic effects of the aforementioned acquired immune mediated pathway.

\subsection{Depression-like behavior in $\mathrm{Mrl} / \mathrm{lpr}$ mice}

MRL lymphoproliferation (MRL/lpr) strain mice develop a systemic autoimmune disease with arthritis, nephritis and neurological-psychiatric disease similar to SLE in humans. The mice show progressive lymphadenopathy due to the accumulation of double negative CD4-8-B220+ alpha beta ${ }^{+} \mathrm{T}$ cells. The underlying defect involves the expression of a functionally inactive Fas receptor, which physiologically promotes activation induced cell death. Prior to the stage at which autoantibodies against DNA and nucleoproteins become detectable, MRL/lpr mice develop depression-like behavior including reduced activity in the forced swim-test and reduced preference for sweet solutions in the two-bottle sweet versus water test. (for review see: (Gulinello and Putterman, 2011)). Thus 
studies on the pathophysiology of depression-like disease in the MRL/lpr autoimmune mouse offers potential new insights into the understanding of depression-like symptoms in SLE patients.

\subsection{Activation of CD40 leads to depression-like behavior in mice}

To study the aetio-pathophysiology of depression-like behavior in autoimmune diseases, we have developed a new mouse model, which is based on immune activation by CD40L-CD40 interactions. The CD40 ligand (CD40L) - CD40 pathway is essential for the autoimmune response to self-antigens (Gast et al., 2013). Mice with neutralization of this ligand-receptor pathway are protected from experimental autoimmune diseases such as experimental autoimmune encephalitis (EAE). Moreover patients with depression have increased plasma levels of soluble CD40 and CD40L (Leo et al., 2006; Neubauer et al., 2013). CD40L is expressed mainly by activated CD4+ T cells and binds to CD40 expressed on macrophages, B-lymphocytes and dendritic cells. CD40 activation by agonistic anti-CD40 monoclonal antibodies (mAb) induces synthesis of chemokines and cytokines including TNF, IL-18 and IFN type I and II. Immune activation is associated with multiorgan inflammation with severe necrotizing hepatitis, lymphadenopathy, splenomegaly, and depression-like behavior in the form of weight loss, decreased feeding, decreased activity and increased NREM sleep (Cathomas et al., 2015; Gast et al., 2013; Taraborrelli et al., 2011). These depression-like behaviors persist up to day 3. Furthermore, CD40 Ab also led to decreased operant motivation for and consumption of sweet-tasting saccharin solution up to day 7 and decreased fear conditioning of a tone to electroshock at day 5, indicating induction of reduced interest in reward and impaired learning/plasticity, respectively (Cathomas et al., 2015). These behavioral effects were coincident with increases in TNF as well as kynurenine and its downstream catabolites in plasma and brain up to days 7-8. Co-injection of the TNF blocker etanercept with CD40 Ab prevented weight loss, decreased activity, increased sleep and reduced interest in reward, and markedly attenuated kynurenine pathway activation in plasma and brain. However, co-injection of an inhibitor of indoleamine 2-3, dioxygenase, the enzyme primarily responsible for tryptophan-to-kynurenine conversion, was without effect on either weight loss 
or saccharin drinking (Cathomas et al., 2015). According to this mouse model, activation of TNF is necessary for causation of depression-like behavior in autoimmune disease. Extrapolating the findings to human suggests that symptoms such as decreased appetite, loss of interest-pleasure and cognitive impairments that are highly comorbid with autoimmine disorder have an aetiopathophysiology that begins with CD40-CD40L-TNF activation, whilst downstream factors remain to be elucidated.

\section{Abnormal clock gene system in autoimmune diseases and depression}

Circadian rhythms are mediated by clock genes, and thereby regulate metabolism and sleep-wake behavior (Eckel-Mahan and Sassone-Corsi, 2013; Franken and Dijk, 2009). The main transcription factors coordinating the circadian rhythms are CLOCK and BMAL1, which form heterodimers and activate expression of Period (Per), Cryptochrome (Cry) and of various clock controlled genes by binding to E-box motives (Dibner et al., 2010). Furthermore, CLOCK can be substituted by its paralog NPAS2 (Reick et al., 2001). The binding of CLOCK:BMAL1 to the E-box is regulated by positive and negative feedback mechanisms (Dibner et al., 2010). Patients with depression show sleep disturbances and an abnormal circadian regulation of hormones including cortisol and melatonin (Parry et al., 2008). Abnormal rhythms of expression of clock genes, due to effects of cytokines on transcription and posttranslational processes including chromatin remodelling, may play a role in depression. Increased risk for developing depression has been identified in individuals with polymorphisms carrying the CC genotype in Cry1 rs2287161 and the TT genotype in Tef rs738499 (Soria et al., 2010). An analysis of postmortem brain tissues from 55 normal controls and 34 patients with depression shows, in the patients, an abnormal phasing of circadian gene expression and potentially disrupted phase relationships between individual circadian genes (Li et al., 2013). Also, the cyclic patterns were much weaker in the depression patients.

Recent studies point to communication of the body clock and the immune system. For example the macrophage response to Salmonella is decreased in mice with an inactive clock gene (Bellet et al., 2013), and the extent of secretion of TNF and 
IL-6 by LPS stimulated macrophages follows a circadian rhythm (Keller et al., 2009). Besides effects of the circadian system on the immune response, the immune system also influences the circadian clock. TNF inhibits the expression of all three Period genes, of Cry-1 and -2 and of the PAR-bZip transcription factors Dbp, Tef and Hlf (Cavadini et al., 2007; Petrzilka et al., 2009). At least some of these effects are due to an interference with E-box-dependent transcription (Cavadini et al., 2007). Both TNF induced Twist1 expression and TNF-mediated inhibition of the cold-inducible RNA binding protein (CIRBP) are involved in immune mediated dysregulation of clock genes (Lopez et al., 2014; Meier et al., 2015). In light of the abnormal clock gene expression in depression, the polymorphisms in the Cry-1 and Tef genes associated with depression, and the dysregulated expression of clock genes in cells exposed to TNF and IL-1 $\beta$, we hypothesise that depression in autoimmune diseases may involve cytokine induced dysregulation of expression of clock genes. The hypothesis is supported by recent findings of abnormal expression of clock genes in rheumatoid arthritis, known for its high depression comorbidity (see above). When arthritis is induced in C57BL/6 mice by i.p. injection of anti-type II collagen mAb on day 1 and LPS (50 $\mu \mathrm{g}$ ) on day 2, the expression of Bmal1, Per2 and Dbp were decreased in the $24 \mathrm{hr}$ cycle in spleen and the joints (Hashiramoto et al., 2010). When analyzing clock genes in cultured human rheumatoid synovial cells treated with TNF, suppression of the expression of Per2, Dbp, Tef and Hlf was found (Yoshida et al., 2013). Recently, a loss of circadian rhythmicity in the expression of the clock genes Per2 and Per3 in CD14+ monocytes was identified in postmenopausal patients with rheumatoid arthritis (Spies et al., 2015). A down regulation of the aforementioned genes was also observed in a transcriptome analysis of genes expressed in the colon mucosa of patients with inflammatory bowel disease (Palmieri et al., 2015). The topic of clock genes, depression and autoimmune diseases has just started to become interesting. From first studies we conclude that the circadian expression of clock genes in autoimmune diseases is altered. It remains to be seen whether the defect is associated with behavioral abnormalities and/or abnormal immune regulation.

\section{Concluding remarks}


The link between immune activation and depression-like behavior becomes evident from studies on injection of cytokines and their triggers such as LPS, PolyI:C and CD40 mAb in rodents. Autoimmune animal models, e.g. MRL/lpr mice or mice treated with agonistic CD40 mAb, support the hypothesis that immune effector pathways cause depression-like behavior. From the studies in SLE and RA outlined above, it can be concluded that in addition to organ specific symptoms arising due to autoimmune inflammation, depression or depressionlike symptoms are also a severe complication, which impairs quality of life. The reports on associations of immune activation with depression-like symptoms point to multiple underlying pathways impacting on these psychological states. Cytokines, autoantibodies and complement factors have all been shown to alter neuronal activity, either directly, by modulating the production of glutamate by microglia, or, in the case of TNF, by enhancing neuronal activity via activation of TNF receptors on astrocytes (Habbas et al., 2015; Piani et al., 1991). When taking one distinct immune effector pathway such as proinflammatory cytokines, their involvement in depression-like symptoms may not be clearly evident because other mechanisms e.g. in SLE, anti-NMDA receptor antibodies or anti-ribosomal $\mathrm{P}$ antibodies, may co-occur and, in contrast to cytokines, the production of autoantibodies may continue into the remission phase. In addition, the consequences of effects of cytokines and autoantibodies on brain circuitries may perpetuate beyond the active disease phase and cause persistence of depressionlike symptoms in an autonomic fashion in the absence of the triggering immune effector pathways. In addition to immunological mechanisms, many other variables including organ dysfunction (eg nephritis and hypertension in SLE), treatment including IFN type I and corticosteroids, social factors, functional disability and coping strategies may be confounding in studies aimed at demonstrating a (causal) relationship between immune activation and depression-like symptoms. Whilst it remains to be determined to what extent psychosocial-stress induced depression symptoms and depression-like symptoms in autoimmune diseases have common or separate pathophysiologies, it is clear that mood, motivation, fatigue and cognition are frequently disrupted 
in autoimmune disorders, constitute a major component of the disease, and need to be the focus of research efforts designed to improve their treatment.

\section{Legend to Figures}

\section{Figure 1}

Cytokines and the clock. Alterations of the circadian system may play a pivotal role in depressive-like behaviour. Cytokines interfere with the physiologic circadian oscillation of clock genes in the suprachiasmatic nucleus of the brain, which receives signals via the light-dark cycle. The oscillation of the peripheral clock is altered in autoimmune diseases in various organs including the liver and colon in hepatitis and colitis, respectively. This may cause abnormal expression of circadian genes. The clock system is also abnormal in lymphoid organs including the spleen and bone marrow. Thereby immune mediated effector pathways may be impaired.

\section{Figure 2}

Impaired expression of clock genes due to effects of proinflammatory cytokines. TNF and IL-1b decrease high amplitude expression of clock genes by inhibiting transcription of the cold-inducible RNA binding protein (CIRBP). Inhibition of CIRBP expression involves NF-kB activation. CIRBP enhances NF-kB expression and thereby eg. regulates TNF mediated apoptosis. In addition to CIRBP, Twist1 is also involved in altering clock gene expression. TNF and IL-1b induce Twist1, which interferes with the activation of E-box mediated transcription by CLOCK-BMAL1. This effect leads to impaired expression of the E-Box regulated clock genes Period-1, -2 and -3 , the cryptochrome genes Cry- 1 and-2, and 
the PAR-bZip transcription factors Dbp, Tef and Hlf. However, recent studies on the expression of clock genes in colon and inflammatory monocytes of mice with DSS induced colitis do not support the in vitro findings of Twist 1 to be involved in suppression of clock genes (Seles C., Strauss L, Rambousek L. and Fontana A. , manuscript in preparation).

\section{Figure 3}

Multiple pathways may lead to depression-like behavior in autoimmune diseases. Immune mediated tissue damage may lead to depression due to the development of hypoxia in heart and pulmonary failure, or due to the development of hypertension in kidney diseases. Depression is also caused by medication used to treat autoimmune diseases, such as treatment with interferon type 1 in multiple sclerosis or corticosteroids in systemic lupus erythematosus. Recognition of self-antigens leads to activation of microglia and macrophages, and of astrocytes, which produce cytokines acting in an autocrine and paracrine manner. This step is followed by the release of molecules including excitatory amino acids, nitric oxide and reactive oxygen species, which alter synaptic signalling pathways and e.g. cause NMDA mediated synaptic activation. The latter effect is enhanced by impaired astrocyte glutamate detoxification. Neurotoxicity is amplified by oxidative stress and toxic effects of TNF on one hand, and poor neurotrophic support on the other. Autoantibodies to NMDA receptors and ribosomal P protein as detected in SLE may contribute to neuronal damage. 


\section{Acknowledgments}

The work was supported by the Swiss National Science Foundation (Project 141055), the Baugarten Stiftung, the Lotex Foundation and the Swiss Multiple

Sclerosis Society to A.F., and the Swiss National Science Foundation (Project 141137) to C.R.P. Adriano Fontana is Hertie Senior Research Professor for Neuroscience of the Gemeinnützige Hertie-Stiftung.

\section{References}

Ahola Kohut S, Williams TS, Jayanthikumar J, Landolt-Marticorena C, Lefebvre A, Silverman E, Levy DM (2013) Depressive symptoms are prevalent in childhood-onset systemic lupus erythematosus (cSLE). Lupus 22, 712-720.

Bachen EA, Chesney MA, Criswell LA (2009) Prevalence of mood and anxiety disorders in women with systemic lupus erythematosus. Arthritis Rheum. 61, 822-829.

Bellet MM, Deriu E, Liu JZ, Grimaldi B, Blaschitz C, Zeller M, Edwards RA, Sahar S, Dandekar S, Baldi P, George MD, Raffatellu M, Sassone-Corsi P (2013) Circadian clock regulates the host response to Salmonella. Proc. Natl. Acad. Sci. U. S. A. 110, 9897-9902.

Bravo-Zehnder M, Toledo EM, Segovia-Miranda F, Serrano FG, Benito MJ, Metz C, Retamal C, Alvarez A, Massardo L, Inestrosa NC, Gonzalez A (2015) Antiribosomal $P$ protein autoantibodies from patients with neuropsychiatric lupus impair memory in mice. Arthritis \& Rheumatology 67, 204-214.

Calderon J, Flores P, Babul M, Aguirre JM, Slachevsky A, Padilla O, Scoriels L, Henriquez C, Carcamo C, Bravo-Zehnder M, Gonzalez A, Massardo L (2014) Systemic lupus erythematosus impairs memory cognitive tests not affected by depression. Lupus 23, 1042-1053.

Cathomas F, Fuertig R, Sigrist H, Newman G, Hoop V, Bizzozzero M, Mueller A, Ceci A, Hengerer B, Seifritz E, Fontana A, Pryce CR (2015) CD40-induced inflammation in mice leads to sustained emotional and cognitive dysfunctions coincident with altered tryptophan metabolsim: a model for depressionautoimmune disease comorbidity. Brain. Behav. Immun. 50, 125-140.

Cavadini G, Petrzilka S, Kohler P, Jud C, Tobler I, Birchler T, Fontana A (2007) TNF-a suppresses the expression of clock genes by interfering with E-boxmediated transcription. Proc. Natl. Acad. Sci. U. S. A. 104, 12843-12848.

Chang EH, Volpe BT, Mackay M, Aranow C, Watson P, Kowal C, Storbeck J, Mattis P, Berlin R, Chen H-I, Mader S, Huerta TS, Huerta PT, Diamond B (2015) Selective impairment of spatial cognition caused by autoantibodies to the Nmethyl-D-aspartate receptor. EBioMedicine 2, 755-764.

Dantzer R, O'Connor JC, Freund GG, Johnson RW, Kelley KW (2008) From inflammation to sickness and depression: when the immune system subjugates the brain. Nature Rev Neurosci 9, 46-57. 
Dibner C, Schibler U, Albrecht U (2010) The mammalian circadian timing system: organization and coordination of central and peripheral clocks. Annu. Rev. Physiol. 72, 517-549.

Dowlati Y, Herrmann N, Swardfager W, Liu H, Sham L, Reim EK, Lanctot KL (2010) A meta-analysis of cytokines in major depression. Biol Psychiatry 67, 446-457.

Eckel-Mahan K, Sassone-Corsi P (2013) Metabolsim and the circadian clock converge. Physiol. Rev. 93, 107-135.

Felger JC, Lotrich FE (2013) Inflammatory cytokines in depression: neurobiological mechanisms and therapeutic implications. Neuroscience 246, 199-229.

Fifield J, Reisine S, Sheehan TJ, McQuillan J (1996) Gender, paid work, and symptoms of emotional distress in rheumatoid arthritis patients. Arthritis Rheum. 39, 427-435.

Franken P, Dijk D-J (2009) Circadian clock genes and sleep homeostasis. Eur. J. Neurosci. 29, 1820-1829.

Fu X, Zunich SM, O'Connor JC, Kavelaars A, Dantzer R, Kelley KW (2010) Central administration of lipopolysaccharide induces depressive-like behavior in vivo and activates brain indoleamine 2,3 dioxygenase in murine organotypic hippocampal slice cultures. Journal of Neuroinflammation 7:43, 1-12.

Gast H, Müller A, Lopez M, Meier D, Huber R, Dechent F, Prinz M, Emmenegger Y, Franken P, Birchler T, Fontana A (2013) CD40 activation induces NREM sleep and modulates genes associated with sleep homeostasis. Brain. Behav. Immun. 27, 133-144.

Gono T, Kawaguchi Y, Yamanaka H (2013) Discoveries in the pathophysiology of neuropsychiatric lupus erythematosus: consequences for therapy. BMC Medicine 11:91. doi: 10.1186.

Greco CM, Li T, Sattar A, Kao AH, Danchenko N, Edmundowicz D, Sutton-Tyrrell K, Tracy RP, Kuller LH, Manzi S (2012) Association between depression and vascular disease in systemic lipus erythematosus. The Journal of Rheumatology 39, 262-268.

Greenley RN, Hommel KA, Nebel J, Raboin T, Li S-H, Simpson P, Mackner L (2010) A meta-analytic review of the psychosocial adjustment of youth with inflammatory bowel disease. J. Pediatr. Psychol. 35, 857-869.

Gulinello M, Putterman C (2011) The MRL/lpr mouse strain as a model for neuropsychiatric systemic lupus erythematosus. Journal of Biomedicine and Biotechnology 2011:207504, 1-15.

Habbas S, Santello M, Becker D, Stubbe H, Zappia G, Liaudet N, Klaus F, Kollias G, Fontana A, Pryce CR, Suter T, Volterra A (2015) Neuroinflammatory TNFa impairs memory via astrocyte signaling. Cell 110.

Haroon E, Raison CL, Miller AH (2012) Psychoneuroimmunology meets neuropsychopharmacology: translational implications of the impact of inflammation on behavior. Neuropsychopharmacology 37, 137-162.

Hashiramoto A, Yamane T, Tsumiyama K, Yoshida K, Komai K, Yamada H, Yamazaki F, Doi M, Okamura H, Shiozawa S (2010) Mammalian clock gene crytochrome regulates arthritis via proinflammatory cytokine TNF-alpha. J. Immunol. 184, 1560-1565. 
Hider SL, Tanveer W, Brownfield A, Mattey DL, Packham JC (2009) Depression in RA patients treated with anti-TNF is common and under-recognized in the rheumatology clinic Rheumatology (Oxford). 48, 1152-1154.

Imeri L, Opp MR (2009) How (and why) the immune system makes us sleep. Nature Reviews Neuroscience 10, 199-210.

Imran MY, Khan SEA, Ahmad NM, Raja SF, Saeed MA, Haider II (2015) Depression in rheumatoid arthritis and its relation to disease activity. Pakistan Journal of Medical Science 31, 393-397.

Isik A, Koca SS, Ozturk A, Mermi O (2007) Anxiety and depression in patients with rheumatoid arthritis. Clin. Rheumatol. 26, 872-878.

Karassa FB, Magliano M, Isenberg DA (2003) Suicide attempts in patients with systemic lupus erythematosus. Annals of Rheumatoid Disorders 62, 58-60.

Karimifar M, Sharifi I, Shafiey K (2013) Anti-ribosomal P antibodies related to depression in early clinical course of systemic lupus erythematosus. Journal of Research in Medical Sciences 18, 860-864.

Karol DE, Criscione-Schreiber LG, Lin M, Clowse MEB (2013) Depressive symptoms and associated factors in systemic lupus erythematosus. Psychosomatics 54, 443-450.

Katz PP, Yelin EH (2001) Activity loss and the onset of depressive symptoms: Do some activities matter more than others? Arthritis Rheum. 44, 1194-1202.

Katzav A, Ben-Ziv T, Blank M, Pick CG, Shoenfeld Y, Chapman J (2014) Antibodyspecific behavioral effects: intracerebroventricular injection of antiphospholipid antibodies induces hyperactive behavior while antiribosomal-P antibodies induces depression and smell deficits in mice. J. Neuroimmunol. 272, 10-15.

Keller M, Mazuch J, Abraham U, Eom GD, Herzog ED, Volk H-D, Kramer A, Maier B (2009) A circadian clock in macrophages controls inflammatory immune responses. Proc. Natl. Acad. Sci. U. S. A. 106, 21407-21412.

Kellner ES, Lee PY, Li Y, Switanek J, Zhuang H, Segal MS, Sobel ES, Satoh M, Reeves WH (2010) Endogenous type-I interferon activity is not associated with depression or fatigue in systemic lupus erythematosus. J. Neuroimmunol. 223, 13-19.

Kivity S, Katzav A, Arango MT, Landau-Rabi M, Zafrir Y, Agmon-Levin N, Blank M, Anaya J-M, Mozes E, Chapman J, Shoenfeld Y (2013) 16/6-idiotype expressing antibodies induce brain inflammation and cognitive impairment in mice: the mosaic of central nervous system involvement in lupus. BMC Medicine 11:90. doi: 10.1186.

Knight A, Weiss P, Morales K, Gerdes M, Rearson M, Vickery M, Keren R (2015) Identifying differences in risk factors for depression and anxiety in pediatric chronic disease: a matched cross-sectional study of youth with lupus/mixed connective tissue disease and their peers with diabetes. The Journal of Pediatrics 167, 1397-1403.

Knight A, Weiss PM, Morales K, Gerdes M, Gutstein A, Vickery M, Keren R (2014) Depression and anxiety and their association with healthcare utilization in pediatric lupus and mixed connective tissue disease patients: a cross-sectional study. Pediatric Rheumatology 12:42.

Krueger JM (2008) The role of cytokines in sleep regulation. Curr. Pharm. Des. 14, 3408-3416. 
Leo R, Di Lorenzo G, Tesauro M, Razzini C, Forleo GB, Chiricolo G, Cola C, Zanasi M, Troisi A, Siracusano A, Lauro R, Romeo F (2006) Association between enhanced soluble CD40 ligand and proinflammatory and prothrombotic states in major depressive disorder: pilot observations on the effects of selective serotonin reuptake inhibitor therapy. J. Clin. Psychiatry 67, 1760-1766.

Li S-X, Liu L-J, Xu L-Z, Gao L, Wang X-F, Zhang J-T, Lu L (2013) Diurnal alterations in circadian genes and peptides in major depressive disorder before and after escitalopram treatment. Psychoneuroendocrinology 38, 2789-2799.

Lopez M, Meier D, Müller A, Franken P, Fujita J, Fontana A (2014) Tumor necrosis factor and transforming growth factor $\mathrm{B}$ regulate clock genes by controlling the expression of the cold inducible RNA-binding protein (CIRBP). J. Biol. Chem. 289, 2736-2744.

Mak A, Tang CS, Ho RC (2013) Serum tumour necrosis factor-alpha is associated with poor health-related quality of life and depresive symptoms in patients with systemic lupus erythematosus. Lupus 22, 254-261.

Makay B, Emiroglu N, Ünsal E (2010) Depression and anxiety in children and adolescents with familial Mediterranean fever. Clin. Rheumatol. 29, 375-379.

Margaretten M, Julian L, Katz P, Yelin E (2011) Depression in patients with rheumatoid arthritis: description, causes and mechanisms. International Journal of Clinical Rheumatology 6, 617-623.

Marrie RA, Reider N, Cohen JY, Stuve O, Sorensen PS, Cutter G, Reingold SC, Trojano M (2015) A systematic review of the incidence and prevalence of autoimmune disease in multiple sclerosis. Multiple Sclerosis Journal 21, 282293.

Meier D, Lopez M, Franken P, Fontana A (2015) Twist1 is a TNF-inducible inhibitor of Clock mediated activation of period genes. PLoS One 10, e0137229

Miller AH, Maletic V, Raison CL (2009) Inflammation and its discontents: the role of cytokines in the pathophysiology of major depression. Biol Psychiatry 65, 732-741.

Mok CC, Chan KL, Cheung EFC, Yip PSF (2014) Suicidal ideation in patients with systemic lupus erythematosus: incidence and risk factors. Rheumatology (Oxford). 53, 714-721.

Neubauer H, Petrak F, Zahn D, Pepinghege F, Hägele A-K, Pirkl P-A, Uhl I, Juckel G, Mügge A, Herpertz S (2013) Newly diagnosed depression is associated with increased beta-thromboglobulin levels and increased expression of platelet activation markers and platelet derived CD40-CD40L. J. Psychiatr. Res. 47, 865-871.

Palagini L, Mosca M, Tani C, Gemignani A, Mauri M, Bombardieri S (2013) Depression and systemic lupus erythematosus: a systematic review. Lupus 22, 409-416.

Palagini L, Tani C, Bruno RM, Gemignani A, Mauri M, Bombardieri S, Riemann D, Mosca M (2014) Poor sleep quality in systemic lupus erythematosus: does it depend on depressive symptoms? Lupus 23, 1350-1357.

Palmieri O, Mazzoccoli G, Bossa F, Maglietta R, Palumbo O, Ancona N, Corritore G, Latiano T, Martino G, Rubino R, Biscaglia G, Scimeca D, Carella M, Annese V, Andriulli A, Latiano A (2015) Systematic analysis of circadian genes using 
genome-wide cDNA microarrays in the inflammatory bowel disease transcriptome. Chronobiol. Int. 32, 903-916.

Panopalis P, Yazdany J, Zell Gillis J, Julian L, Trupin L, Hersh AO, Criswell LA, Katz P, Yelin E (2008) Health care costs and costs associated with changes in work productivity among persons with systemic lupus erythematosus. Arthritis Rheum. 59, 1788-1795.

Parry BL, Meliska CJ, Sorenson DL, Lopez AM, Martinez LF, Nowakowski S, Elliott JA, Hauger RL, Kripke DF (2008) Plasma melatonin circadian rhythm disturbances during pregnancy and postpartum in depressed women and women with personal or family histories of depression. A. J. Psychiatry 165, 1551-1558.

Petrzilka S, Taraborrelli C, Cavadini G, Fontana A, Birchler T (2009) Clock gene modulation by TNF-alpha depends on calcium and p38 MAP kinase signaling. J. Biol. Rhythms 24, 283-294.

Pettersson S, Lövgren M, Eriksson LE, Moberg C, Svenungsson E, Gunnarsson I, Welin Henriksson E (2012) An exploration of patient-reported symptoms in systemic lupus erythematosus and the relationship to health-related quality of life. Scand. J. Rheumatol. 41, 383-390.

Piani D, Frei K, Cuenod M, Fontana A (1991) Murine brain macrophages induced NMDA receptor mediated neurotoxicity in vitro by secreting glutamate. Neurosci. Lett. 133, 159-162.

Raison CL, Rutherford RE, Woolwine BJ, Shuo C, Schettler P, Drake DF, Haroon E, Miller AH (2013) A randomized controlled trial of the tumor necrosis factor antagonist infliximab for treatment-resistant depression: the role of baseline inflammatory biomarkers. JAMA Psychiatry 70, 31-41.

Reick M, Garcia JA, Dudley C, McKnight SL (2001) NPAS2: an analog of clock operative in the mammalian forebrain. Science 293, 506-509.

Shelton RC, Claiborne J, Sidoryk-Wegrzynowicz M, Reddy R, Aschner M, Lewis DA, Mirnics K (2011) Altered expression of genes involved in inflammation and apoptosis in frontal cortex in major depression. Mol Psychiatry 16, 751-762.

Shen B, Feng G, Tang W, Huang X, Yan H, He Y, Chen W, Da Z, Liu H, Gu Z (2014) The quality of life in Chinese patients with systemic lupus erythematosus is associated with disease activity and psychiatric disorders: a path analysis. Clin. Exp. Rheumatol. 32, 101-107.

Soria V, Martinez-Amoros E, Escaramis G, Valero J, Perez-Egea R, Garcia C, Gutierrez-Zotes A, Puigdemont D, Bayes M, Crespo JM, Martorell L, Vilella E, Labad A, Vallejo J, Perez V, Menchon JM, Estivill X, Gratacos M, Urretavizcaya M (2010) Differential association of circadian genes with mood disorders: CRY1 and NPAS2 are associated with unipolar major depression and CLOCK and VIP with bipolar disorder. Neuropsychopharmacology 35, 1279-1289.

Spies CM, Hoff P, Mazuch J, Gaber T, Maier B, Strehl C, Hahne M, Jakstadt M, Huscher D, Burmester GR, Detert J, Kramer A, Buttgereit F (2015) Circadian rhythms of cellular immunity in rheumatoid arthritis: a hypothesis-generating study. Clin. Exp. Rheumatol. 33, 34-43.

Taraborrelli C, Palchykova S, Tobler I, Gast H, Birchler T, Fontana A (2011) TNFR1 is essential for CD40, but not for lipopolysaccharide-induced sickness behavior and clock gene dysregulation. Brain Behav Immunity 25, 434-442. 
Tay SH, Cheung PPM, Mak A (2015) Active disease is independently associated with more severe anxiety rather than depressive symptoms in patients with systemic lupus erythematosus. Lupus 24, 1392-1399.

van Exel E, Jacobs J, Korswagen L-A, Voskuyl AE, Stek M, Dekker J, Bultink IEM (2013) Depression in systemic lupus erythematosus, dependent on or independent of severity of disease. Lupus 22, 1462-1469.

Vina ER, Hausmann LRM, Utset TO, Masi CM, Liang KP, Kent Kwoh C (2015) Perceptios of racism in healthcare among patients with systemic lupus erythematosus: a cross-sectional study. Lupus Science \& Medicine 2, e000110.

Waldheim E, Elkan A-C, Pettersson S, van Vollenhoven R, Bergman S, Frostegard J, Welin Henriksson E (2013) Health-related quality of life, fatigue and mood in patients with SLE and high levels of pain compared to controls and patients with low levels of pain. Lupus 22, 1118-1127.

Wang S-L, Chang C-H, Hu L-Y, Tsai S-J, Yang AC, You Z-H (2014) Risk of developing depressive disorders following rheumatoid arthritis: a nationwide population-based study. Plos One 9, e107791.

Xie L-F, Chen P-L, Pan H-F, Taio J-H, Li X-P, Zhang Y-J, Zhai Y, Ye D-Q (2012) Prevalence and correlates of suicidal ideation in SLE inpatients: Chinese experience. Rheumatol. Int. 32, 2707-2714.

Xu J, Cheng YQ, Chen B, Bai R, Li S, Xu XF, Xu L, Wen JF, Lu ZP, Zeng XF (2013) Depression in systemic lupus erythematosus patients is associated with linkpolymorphism but not methylation status of the 5HTT promoter region. Lupus 22, 1001-1010.

Yilmaz-Oner S, Oner C, Mert Dogukan F, Filam Moses T, Demir K, Tekayev N, Atagunduz P, Tuglular S, Direskeneli H (in press) Health-related quality of life assessed by LupusQoL questionnaire and SF-36 in Turkish patients with systemic lupus erythematosus. Clin. Rheumatol.

Yoshida K, Hashiramoto A, Okano T, Yamane T, Shibanuma N, Shiozawa S (2013) TNF-a modulates expression of the circadian clock gene Pers in rheumatoid synovial cells. Scand. J. Rheumatol. 42, 276-280. 